\title{
ПОЛУПОЛЕВЫЕ ПЛОСКОСТИ РАНГА 2, ДОПУСКАЮЩИЕ ГРУППУ $S_{3}{ }^{1}$
}

\section{О. В. Кравцова, Т. В. Моисеенкова}

\begin{abstract}
Одна из классических задач проективной геометрии - построение объекта по известным ограничениям на его автоморфизмы. Рассматриваются конечные проективные плоскости, координатизируемые полуполем, т. е. алгебраической системой, удовлетворяющей аксиомам тела, за исключением ассоциативности умножения. Такая плоскость является плоскостью трансляций и обладает также транзитивной группой элаций с аффинной осью. Пусть $\pi$ - полуполевая плоскость порядка $p^{2 n}$ с ядром, содержащим $G F\left(p^{n}\right)(p-$ простое число), группа линейных автотопизмов которой содержит подгруппу $H$, изоморфную симметрической группе $S_{3}$. Для построения и исследования таких плоскостей применяется подход с использованием линейного пространства и регулярного множества - специального семейства линейных преобразований. Построено матричное представление подгруппы $H$ и регулярного множества полуполевой плоскости для $p=2$ и $p>2$. Изучена возможность присутствия центральных коллинеаций в подгруппе $H$. Показано, что полуполевая плоскость порядка $3^{2 n}$ с ядром $G F\left(3^{n}\right)$ не допускает $S_{3}$ в группе линейных автотопизмов. Найдены примеры полуполевых плоскостей порядков 16 и 625 , допускающих $S_{3}$. Полученные результаты могут быть обобщены на случай полуполевых плоскостей ранга более двух и могут быть использованы, в частности, при исследовании известной гипотезы о разрешимости полной группы коллинеаций конечной недезарговой полуполевой плоскости.
\end{abstract}

Ключевые слова: полуполевая плоскость, группа автотопизмов, симметрическая группа, бэровская инволюция, гомология, регулярное множество.

O. V. Kravtsova, T. V. Moiseenkova. Semifield planes of rank 2 admitting the group $S_{3}$.

One of the classical problems in projective geometry is to construct an object from known constraints on its automorphisms. We consider finite projective planes coordinatized by a semifield, i.e., by an algebraic system satisfying all axioms of a skew-field except for the associativity of multiplication. Such a plane is a translation plane admitting a transitive elation group with an affine axis. Let $\pi$ be a semifield plane of order $p^{2 n}$ with a kernel containing $G F\left(p^{n}\right)$ for prime $p$, and let the linear autotopism group of $\pi$ contain a subgroup $H$ isomorphic to the symmetric group $S_{3}$. For the construction and analysis of such planes, we use a linear space and a spread set, which is a special family of linear mappings. We find a matrix representation for the subgroup $H$ and for the spread set of a semifield plane if $p=2$ and if $p>2$. We also study the existence of central collineations in $H$. It is proved that a semifield plane of order $3^{2 n}$ with kernel $G F\left(3^{n}\right)$ admits no subgroups isomorphic to $S_{3}$ in the linear autotopism group. Examples of semifield planes of order 16 and 625 admitting $S_{3}$ are found. The obtained results can be generalized for semifield planes of rank greater than 2 and can be applied, in particular, for studying the known hypothesis that the full collineation group of any finite non-Desarguesian semifield plane is solvable.

Keywords: semifield plane, autotopism group, symmetric group, Baer involution, homology, spread set.

MSC: 51A35, 51A40, 20B25

DOI: $10.21538 / 0134-4889-2019-25-4-118-128$

\section{Введение}

Координатизация точек и прямых в конечной проективной плоскости устанавливает связь между геометрическими свойствами плоскости и алгебраическими свойствами ее координатизирующего множества. Так, классическая или дезаргова, проективная плоскость координатизируется полем, а плоскость трансляций - квазиполем.

Проективная плоскость называется полуполевой, если ее координатизирующее множество является полуполем (semifield). Такая плоскость одновременно является плоскостью трансляций и дуальна плоскости трансляций. Особенности строения координатизирующего множества

\footnotetext{
${ }^{1}$ Исследование выполнено при финансовой поддержке РФФИ в рамках научного проекта № 19-0100566 A.
} 
вызывают ряд вопросов, связанных со строением полной группы коллинеаций (автоморфизмов) полуполевых плоскостей. Наиболее известна гипотеза [1, вопрос 11.76] о разрешимости полной группы коллинеаций всякой полуполевой недезарговой плоскости конечного порядка. $\mathrm{K}$ настоящему времени эта гипотеза подтверждена лишь для некоторых классов полуполевых плоскостей (см., например, [2]). В связи с этим информация о коллинеациях конечной полуполевой плоскости является важной.

Известен способ задания полуполевой плоскости, как и всякой плоскости трансляций, с использованием линейного пространства и специального семейства линейных преобразований, так называемого регулярного множества. Матричное представление регулярного множества определяет алгебраические свойства координатизирующего полуполя и геометрические свойства полуполевой плоскости, в том числе строение группы коллинеаций. Таким образом, представляет интерес как задача исследования группы коллинеаций при известном представлении регулярного множества, так и обратная задача - построение конечных проективных плоскостей по известным ограничениям на группу коллинеаций (см., например, [3]). Большое значение приобрели результаты [4] о полуполевой плоскости, допускающей бэровскую инволюцию.

Симметрическая группа $S_{3}$ является некоммутативной группой минимального порядка, ее наличие в группе коллинеаций проективной плоскости и в группе автоморфизмов координатизирующего множества может указывать на наличие особенных свойств. Так, среди всех 23 неизоморфных полуполей порядка 16 ровно одно имеет группу автоморфизмов, изоморфную $S_{3}$. Таким же свойством обладает исключительное полуполе Хентзела-Ру́а порядка 64, не являющееся ни лево-, ни правопримитивным [5]. Кроме того, поскольку $S_{3}$ содержится во многих известных группах, условие существования $S_{3}$ в группе автотопизмов приводит к получению важных технических результатов для дальнейших исследований. Отметим также особую роль группы $S_{3}$ в исследовании полуполевых плоскостей в связи с классификацией полуполей не только с точностью до изотопизма, но и с точностью до $S_{3}$ (так называемые орбиты Кнута, см., например, $[6])$.

Пусть $\pi$ - полуполевая плоскость порядка $p^{2 n}$ с ядром порядка $p^{n}$ ( $p-$ простое число), подгруппа линейных автотопизмов (коллинеаций, фиксирующих треугольник) которой содержит подгруппу $H$, изоморфную симметрической группе $S_{3}$. Построено матричное представление подгруппы $H$ в $G L_{4}\left(p^{n}\right)$ и регулярного множества плоскости $\pi$ в $G L_{2}\left(p^{n}\right) \cup\{0\}$. Найдены примеры таких плоскостей порядка 16 и 625.

Основные результаты работы, приведеные в теоремах 1-3, анонсированы в [7].

\section{1. Основные определения и предварительные обсуждения}

Введем кратко основные определения, обозначения и результаты, необходимые для работы. Для подробного ознакомления рекомендуем [8;9].

О п р е д е л е н и е 1 . В соответствии с [8, гл. VIII] полуполем будем называть множество $S$, на котором определены две бинарные алгебраические операции + и $*$, при выполнении условий:

1) $\langle S,+\rangle$ - абелева группа с нейтральным элементом 0 ;

2) $\left\langle S^{*}, *\right\rangle$ - лупа $\left(S^{*}=S \backslash\{0\}\right)$;

3) выполняются дистрибутивные законы $a *(b+c)=a * b+a * c,(b+c) * a=b * a+c * a$ для любых $a, b, c \in S$.

Полуполе $S$ содержит подмножества $N_{r}, N_{m}, N_{l}$, называемые правым, средним и левым ядрами соответственно:

$$
\begin{aligned}
N_{r} & =\{n \in S \mid(a * b) * n=a *(b * n) \forall a, b \in S\}, \\
N_{m} & =\{n \in S \mid(a * n) * b=a *(n * b) \forall a, b \in S\}, \\
N_{l} & =\{n \in S \mid n *(a * b)=n *(a * b) \forall a, b \in S\},
\end{aligned}
$$


пересечение $N_{l} \cap N_{m} \cap N_{r}$ называют ядром полуполя. Ядра конечного полуполя являются подполями, и полуполе можно рассматривать как линейное пространство над каждым из них. Следовательно, порядок конечного полуполя равен степени простого числа.

Рассмотрим линейное пространство $W$ размерности $d$ над полем $F \simeq G F\left(p^{n}\right)$ и инъективное отображение $\theta: W \rightarrow G L_{d}\left(p^{n}\right) \cup\{0\}$, удовлетворяющее условиям:

1) образом нулевого вектора является нулевая матрица;

2) единичная матрица $E$ имеет прообраз в $W$;

2) $\theta(x+y)=\theta(x)+\theta(y)$ для всех $x, y \in W$.

Определим на $W$ умножение правилом $x * y=x \cdot \theta(y)$, тогда $\langle W,+, *\rangle$ - полуполе порядка $p^{n d}$. Множество матриц $R=\{\theta(y) \mid y \in W\}$ называют при этом регулярным множеством (spread set, см. [8, гл. VII]). Рассмотрим внешнюю прямую сумму $V=W \oplus W$ и определим проективную плоскость $\pi$ следующим образом:

1 ) элементы $(x, y)$, где $x, y \in W$, пространства $V$ назовем аффинными точками плоскости $\pi$;

2) аффинными прямыми назовем смежные классы по подгруппам

$$
\begin{aligned}
V(m) & =\{(x, x \theta(m)) \mid x \in W\}, \quad m \in W \\
V(\infty) & =\{(0, y) \mid y \in W\}
\end{aligned}
$$

3) множество всех смежных классов по одной подгруппе $V(m)$ или $V(\infty)$ назовем особой точкой $(m)$ или $(\infty)$ соответственно;

4) множество особых точек назовем особой прямой [ $\infty]$

5) инцидентность определим теоретико-множественным включением.

Построенная таким образом проективная плоскость является полуполевой плоскостью порядка $p^{n d}$, число $d$ будем называть ее рангом.

Полная группа коллинеаций полуполевой плоскости содержит подгруппу автотопизмов $\Lambda$, т. е. коллинеаций, фиксирующих треугольник с вершинами $(0,0),(0),(\infty)$ и сторонами $[0,0]$, $[0],[\infty]$. Автотопизмы задаются полулинейными преобразованиями пространства $V$ и определяются блочно-диагональными матрицами,

$$
(x, y)^{\lambda}=\left(x^{\sigma}, y^{\sigma}\right)\left(\begin{array}{cc}
A & 0 \\
0 & B
\end{array}\right)
$$

здесь $\sigma$ - автоморфизм основного поля $F$, действующий на координаты вектора; $A, B \in$ $G L_{d}\left(p^{n}\right)$. При этом сохранение инцидентности требует выполнения условия

$$
A^{-1}[\theta(m)]^{\sigma} B \in R \quad \forall \theta(m) \in R .
$$

Обозначим через $\Lambda_{0}$ подгруппу линейных автотопизмов $(\sigma=1), \Lambda / \Lambda_{0} \subset \operatorname{Aut}(F)$,

$$
A^{-1} \theta(m) B \in R \quad \forall \theta(m) \in R .
$$

Мы рассматриваем полуполевые плоскости ранга 2 над полем $F \simeq G F\left(p^{n}\right)$, считая, что ядро полуполя либо совпадает с $F$, либо содержит $F$ (в этом случае плоскость дезаргова, $R$ - поле). Группа линейных автотопизмов состоит из матриц размерности $4 \times 4$ с элементами из $F$, регулярное множество $R$ содержится в $G L_{2}\left(p^{n}\right) \cup\{0\}$. Пусть $H<\Lambda_{0}, H=\langle\tau, \sigma\rangle \simeq S_{3}$, где $\tau$ и $\sigma$ - неперестановочные инволюции, коллинеация $\gamma=\tau \sigma$ имеет порядок 3. Обсудим прежде всего геометрический смысл элементов группы $H$. Как известно, всякая коллинеация порядка два проективной плоскости является либо центральной, либо бэровской коллинеацией [8, теорема 4.3].

О п р е д е л е н и е 2 . Коллинеация проективной плоскости $\pi$ порядка $m$ называется бэровской, если она фиксирует поточечно максимальную подплоскость порядка $\sqrt{|\pi|}=\sqrt{m}$ (бэровскую подплоскость). 
О п р е д е л е н и е 3 . Коллинеация проективной плоскости $\pi$ называется центральной (или перспективностью), если она фиксирует поточечно некоторую прямую (ось), некоторую точку (центр) и все прямые, проходящие через центр (не поточечно). Если центр инцидентен оси, то коллинеация называется элацией, в противном случае - гомологией. Если $m-$ порядок проективной плоскости, то $m$ делится на порядок всякой элации, $m-1$ делится на порядок всякой гомологии.

Центральные коллинеации образуют в группе автотопизмов следующие циклические подгруппы [2]:

1) $H_{r} \simeq N_{r}^{*}$ - группа гомологий с осью $[0,0]$ и центром $(\infty)$;

2) $H_{l} \simeq N_{l}^{*}$ - группа гомологий с осью [ $\left.\infty\right]$ и центром $(0,0)$;

3) $H_{m} \simeq N_{m}^{*}$ - группа гомологий с осью [0] и центром (0).

При этом

$$
\begin{gathered}
H_{r}=\left\{\left(\begin{array}{cc}
E & 0 \\
0 & A
\end{array}\right) \mid \theta(m) A \in R \forall \theta(m) \in R\right\}, \\
H_{m}=\left\{\left(\begin{array}{cc}
A & 0 \\
0 & E
\end{array}\right) \mid A \theta(m) \in R \forall \theta(m) \in R\right\}, \\
H_{l}=\left\{\left(\begin{array}{ll}
A & 0 \\
0 & A
\end{array}\right) \mid \theta(m) A=A \theta(m) \forall \theta(m) \in R\right\} .
\end{gathered}
$$

Таким образом, если порядок рассматриваемой полуполевой плоскости $\pi$ равен $p^{2 n}$, то при $p=2$ инволюции $\tau$ и $\sigma$ могут быть только бэровскими, при $p>2-$ бэровскими коллинеациями либо гомологиями, произведение $\tau \sigma$ может быть гомологией при $p \neq 3$. Случаи $p=2, p=3$ и $p>3$ требуют отдельного изучения.

\section{2. Случай $p=2$}

Теорема 1. Пусть $\pi$ - полуполевая плоскость порядка $2^{2 n}$ с ядром, содержащим поле $F \simeq G F\left(2^{n}\right)$, группа линейных автотопизмов которой содержит подгруппу $H$, изоморфную симметрической группе $S_{3}$. Тогда базис 4-мерного линейного пространства над $F$ может быть выбран так, что регулярное множество плоскости в $G L_{2}(F) \cup\{0\}$ имеет вид

$$
R=\left\{\theta(v, u)=\left(\begin{array}{cc}
u+v+m(v) & f(v)+m(u) \\
v & u
\end{array}\right) \mid u, v \in F\right\}
$$

где $m, f$ - аддитивные функиии на $F$, причем $f$ взаимнооднозначна и $m(1)=0$. Кроме того,

1) если $H$ содержит гомологию с осъю $[0,0]$ и иентром $(\infty), \operatorname{mo~} f(x)=x \forall x \in F$;

2) если $H$ содержсит гомологию с осъю [0] и центром (0), mо $f(x)=m(m(x))+m(x)+x$ $\forall x \in F$;

3) если $H$ содержит гомологию с осъю [ [] и центром $(0,0), \operatorname{mo} f(x)=x, m(x)=0 \forall x \in F$, плоскость дезаргова;

4) если $Н$ не содержит гомологий, то функции $m$ и $f$ удовлетворяют условиям

$$
m(m(x))=m(x), \quad f(m(x))=m(x), \quad m(f(x))=m(x)+f(x)+x, \quad f(f(x))=x \quad \forall x \in F .
$$

Д о к а з а т е л ь с т в о. Так как $|\pi|=2^{2 n}$, элементы $\tau$ и $\sigma$ являются бэровскими инволюциями. Воспользуемся результатами [4]: если полуполевая плоскость порядка $2^{2 n}$ с ядром порядка $\geq 2^{n}$ допускает бэровскую инволюцию $\tau$ в линейном трансляционном дополнении, то

$$
\tau=\left(\begin{array}{llll}
1 & 1 & 0 & 0 \\
0 & 1 & 0 & 0 \\
0 & 0 & 1 & 1 \\
0 & 0 & 0 & 1
\end{array}\right),
$$


регулярное множество имеет вид (2.1). Положим

$$
L=\left(\begin{array}{ll}
1 & 1 \\
0 & 1
\end{array}\right), \quad \tau=\left(\begin{array}{cc}
L & 0 \\
0 & L
\end{array}\right), \quad \sigma=\left(\begin{array}{cc}
A & 0 \\
0 & B
\end{array}\right), \quad \text { где } A, B \in G L_{2}\left(p^{n}\right) .
$$

Тогда для инволюции $\sigma$ выполнены условия

1) $A^{2}=B^{2}=E$,

2) $A L \neq L A$ или $B L \neq L B$,

3) $(L A)^{3}=(L B)^{3}=E$.

Отсюда матрицы $A$ и $B$ равны либо $L$ (не одновременно), либо матрице вида

$$
\left(\begin{array}{cc}
a & a^{2}+1 \\
1 & a
\end{array}\right), \quad a \in F
$$

Заметим, что верно равенство $\left(\begin{array}{ll}1 & a \\ 0 & 1\end{array}\right)\left(\begin{array}{cc}a & a^{2}+1 \\ 1 & a\end{array}\right)\left(\begin{array}{ll}1 & a \\ 0 & 1\end{array}\right)^{-1}=\left(\begin{array}{ll}0 & 1 \\ 1 & 0\end{array}\right)=M$, поэтому базис линейного пространства можно выбрать так (не меняя $\tau$ ), что

$$
\sigma \in\left\{\left(\begin{array}{cc}
L & 0 \\
0 & M
\end{array}\right),\left(\begin{array}{cc}
M & 0 \\
0 & L
\end{array}\right),\left(\begin{array}{cc}
M & 0 \\
0 & M
\end{array}\right)\right\} .
$$

Рассмотрим подробно все три возможных случая. В первом случае коллинеация $\gamma=\tau \sigma$ определяется матрицей

$$
\gamma=\left(\begin{array}{cc}
E & 0 \\
0 & L M
\end{array}\right)
$$

и является гомологией порядка 3 с осью $[0,0]$ и центром $(\infty)$, при этом матрица $L M$ удовлетворяет условию $\theta(v, u) L M \in R$ для всех $v, u$. При $v=0$ имеем

$$
\theta(0, u) L M=\left(\begin{array}{cc}
u & m(u) \\
0 & u
\end{array}\right)\left(\begin{array}{ll}
1 & 1 \\
1 & 0
\end{array}\right)=\left(\begin{array}{cc}
u+m(u) & u \\
u & 0
\end{array}\right)=\theta(u, 0),
$$

отсюда $f(u)=u$. Рассмотрение $u=0$ не дает новых ограничений на функции $f$ и $m$.

Во втором случае

$$
\gamma=\left(\begin{array}{cc}
L M & 0 \\
0 & E
\end{array}\right)
$$

является гомологией порядка 3 с осью [0] и центром $(0)$, при этом $L M \theta(v, u) \in R$ для всех $v, u$. При $v=0$

$$
\operatorname{LM\theta }(0, u)=\left(\begin{array}{ll}
1 & 1 \\
1 & 0
\end{array}\right)\left(\begin{array}{cc}
u & m(u) \\
0 & u
\end{array}\right)=\left(\begin{array}{cc}
u & m(u)+u \\
u & m(u)
\end{array}\right)=\theta(0, m(u))
$$

отсюда $f(u)=m(m(u))+m(u)+u$. При $u=0$ получим то же условие.

В третьем случае для $A=B=M$ должно выполняться условие (1.1). При $v=0$ имеем

$$
M^{-1} \theta(0, u) M=\left(\begin{array}{cc}
0 & 1 \\
1 & 0
\end{array}\right)\left(\begin{array}{cc}
u & m(u) \\
0 & u
\end{array}\right)\left(\begin{array}{ll}
0 & 1 \\
1 & 0
\end{array}\right)=\left(\begin{array}{cc}
u & 0 \\
m(u) & u
\end{array}\right)=\theta(m(u), u),
$$

поэтому $m(m(x))=m(x), f(m(x))=m(x)$. При $u=0$ получаем

$$
M^{-1} \theta(v, 0) M=\left(\begin{array}{ll}
0 & 1 \\
1 & 0
\end{array}\right)\left(\begin{array}{cc}
v+m(v) & f(v) \\
v & 0
\end{array}\right)\left(\begin{array}{ll}
0 & 1 \\
1 & 0
\end{array}\right)=\left(\begin{array}{cc}
0 & v \\
f(v) & v+m(v)
\end{array}\right)=\theta(f(v), v+m(v)),
$$

поэтому $m(f(x))=m(x)+f(x)+x, f(f(x))=x$. Поскольку отображение $m(x)$ в общем случае не является биективным, полученные условия трудно преобразовать к более удобному виду.

Заметим, что если в третьем случае коллинеация $\gamma$ является гомологией, то ее ось - особая прямая $[\infty]$, центр - точка $(0,0)$, при этом матрица $L M$ должна быть перестановочна со всеми матрицами регулярного множества. Из этого условия $L M \theta(v, u)=\theta(v, u) L M$ получим $f(v)=v, m(v)=0$ для всех $v$, поэтому в силу линейности $m$ и $f$ регулярное множество является полем, т. е. плоскость $\pi$ дезаргова.

Теорема 1 полностью доказана. 


\section{3. Случай $p>2$}

Лемма. Пусть $\pi$ - полуполевая плоскость порлдка $p^{2 n}$ с ядром, содержсащим поле $F \simeq$ $G F\left(p^{n}\right)(p>2$ - простое), группа линейных автотопизмов которой содержит подгруппу $H$, изоморфную симметрической группе $S_{3}$. Тогда инволюиии $\tau, \sigma \in H$ являются бэровскими, базис 4-мерного линейного пространства над F может быть выбран так, что

$$
\tau=\left(\begin{array}{cccc}
-1 & 0 & 0 & 0 \\
0 & 1 & 0 & 0 \\
0 & 0 & -1 & 0 \\
0 & 0 & 0 & 1
\end{array}\right)
$$

регулярное множество плоскости в $G L_{2}(F) \cup\{0\}$ имеет вид

$$
R=\left\{\theta(v, u)=\left(\begin{array}{cc}
m(u) & f(v) \\
v & u
\end{array}\right) \mid u, v \in F\right\}
$$

где $m, f$ - аддитивные взаимнооднозначные функиии на $F$, причем $m(1)=1$. При этом $\sigma=\left(\begin{array}{cc}A & 0 \\ 0 & B\end{array}\right)$, где $A, B \in\{L, M\}$ при $p>3 ; A, B \in\left\{L, A_{1}, A_{2}\right\}$ nри $p=3 ;(A, B) \neq(L, L)$,

$$
L=\left(\begin{array}{rr}
-1 & 0 \\
0 & 1
\end{array}\right), \quad M=\left(\begin{array}{rr}
1 / 2 & 1 / 2 \\
3 / 2 & -1 / 2
\end{array}\right), \quad A_{1}=\left(\begin{array}{rr}
-1 & 0 \\
1 & 1
\end{array}\right), \quad A_{2}=\left(\begin{array}{rr}
-1 & 1 \\
0 & 1
\end{array}\right) .
$$

Д о к а з а т е л ь с т в о. В случае нечетного порядка плоскости $\pi$ центральные коллинеации порядка 2 в группе автотопизмов $\Lambda$ являются гомологиями и определяются матрицами

$$
\left(\begin{array}{cc}
-E & 0 \\
0 & E
\end{array}\right), \quad\left(\begin{array}{cc}
E & 0 \\
0 & -E
\end{array}\right), \quad\left(\begin{array}{cc}
-E & 0 \\
0 & -E
\end{array}\right)
$$

которые не сопряжены в $\Lambda$. Поэтому инволюции $\tau, \sigma$, как и для $p=2$, являются бэровскими коллинеациями. Воспользуемся результатами [10]: в подходящем базисе линейного пространства бэровская инволюция определяется матрицей (3.1), а регулярное множество имеет вид (3.2).

Инволюция $\sigma$ должна удовлетворять условиям 1-3 из доказательства теоремы 1 . При этом для матрицы $A$ (аналогично для $B$ ) возможны четыре ситуации $(a \in F, a \neq 0)$ :

1) $A=L$;2) $p=3$ и $A=\left(\begin{array}{cc}-1 & 0 \\ a & 1\end{array}\right)$; 3) $p=3$ и $A=\left(\begin{array}{cc}-1 & a \\ 0 & 1\end{array}\right)$; 4) $p \neq 3$ и $A=\left(\begin{array}{cc}1 / 2 & a \\ 3 /(4 a) & -1 / 2\end{array}\right)$.

Для случаев 2)-4) можно выбрать замену базиса линейного пространства, упрощающую вид матрицы $A$ и сохраняющую вид $L$. Действительно,

$$
\begin{gathered}
\left(\begin{array}{ll}
a & 0 \\
0 & 1
\end{array}\right)\left(\begin{array}{cc}
-1 & 0 \\
a & 1
\end{array}\right)\left(\begin{array}{cc}
a^{-1} & 0 \\
0 & 1
\end{array}\right)=\left(\begin{array}{cc}
-1 & 0 \\
1 & 1
\end{array}\right)=A_{1}, \\
\left(\begin{array}{ll}
1 & 0 \\
0 & a
\end{array}\right)\left(\begin{array}{cc}
-1 & a \\
0 & 1
\end{array}\right)\left(\begin{array}{cc}
1 & 0 \\
0 & a^{-1}
\end{array}\right)=\left(\begin{array}{cc}
-1 & 1 \\
0 & 1
\end{array}\right)=A_{2}, \\
\left(\begin{array}{cc}
1 & 0 \\
0 & 2 a
\end{array}\right)\left(\begin{array}{cc}
\frac{1}{2} & a \\
3 /(4 a) & -1 / 2
\end{array}\right)\left(\begin{array}{cc}
1 & 0 \\
0 & 1 /(2 a)
\end{array}\right)=\left(\begin{array}{cc}
1 / 2 & 1 / 2 \\
3 / 2 & -1 / 2
\end{array}\right)=M .
\end{gathered}
$$

Лемма доказана.

Теорема 2. Если $\pi$ - полуполевая плоскость порядка $3^{2 n}$ с ядром, содержащим поле $F \simeq G F\left(3^{n}\right)$, то группа линейных над $F$ автотопизмов не содержит подгруппу, изоморфную симметрической группе $S_{3}$. 
Д о к а з а т е л ь с т в о. Если $A=L$ или $B=L$, то $\gamma=\tau \sigma$ является гомологией порядка 3 , что невозможно, так как $|\pi|-1$ не делится на 3.

Для остальных выделенных в лемме случаев при $p=3$ проверим выполнение условия (1.1). Непосредственные расчеты показывают, что

$$
\begin{gathered}
A_{1}^{-1} \theta(v, 0) A_{1}=\left(\begin{array}{cc}
-f(v) & -f(v) \\
-v+f(v) & f(v)
\end{array}\right)=\theta(-v+f(v), f(v)) \Rightarrow f(f(v))=0 \forall v ; \\
A_{2}^{-1} \theta(v, 0) A_{2}=\left(\begin{array}{cc}
-v & v-f(v) \\
-v & v
\end{array}\right)=\theta(-v, v) \Rightarrow f(-v)=v-f(v) \forall v ; \\
A_{1}^{-1} \theta(0, u) A_{2}=\left(\begin{array}{cc}
m(u) & -m(u) \\
-m(u) & m(u)+u
\end{array}\right)=\theta(-m(u), m(u)+u) \Rightarrow m(m(u))=0 \forall u ; \\
A_{2}^{-1} \theta(0, u) A_{1}=\left(\begin{array}{cc}
m(u)+u & u \\
u & u
\end{array}\right)=\theta(u, u) \Rightarrow m(u)=m(u)+u \forall u .
\end{gathered}
$$

Учитывая инъективность отображений $m$ и $f$, заключаем, что все перечисленные случаи невозможны.

Теорема 2 доказана.

Отметим, что частный случай $|\pi|=81$ представлен первым автором в докладе на Международной конференции G2A2 (Екатеринбург, 2015): если полуполевая плоскость порядка 81 допускает бэровскую инволюцию в группе автотопизмов, то порядок группы автотопизмов равен $2^{k}$, где $8 \leq k \leq 11$.

Теорема 3. Пусть $\pi$ - полуполевая плоскость порядка $p^{2 n}$, с ядром, содержащим поле $F \simeq G F\left(p^{n}\right)(p>3$ - простое $)$, группа линейных автотопизмов которой содержст подгруппу $H$, изоморфную симметрической группе $S_{3}$. Тогда базис 4-мерного линейного пространства над $F$ может быть выбран так, что регулярное множество плоскости в $G L_{2}(F) \cup\{0\}$ имеет вид (3.2). Кроме того,

1) если $H$ содержст гомологию с осъю $[0,0]$ и чентром $(\infty), \operatorname{mo} f(x)=-m(x) / 3 \forall x \in F$;

2) если $H$ содержит гомологию с осъю [0] и центром (0), mо $f(m(x))=m(f(x))=-x / 3$ $\forall x \in F$

3) если $H$ содержит гомологию с осъю $[\infty]$ и центром $(0,0)$, то $p-3$ не является квадратом, $f(x)=-x / 3, m(x)=x \forall x \in F$, плоскость дезаргова;

4) если $H$ не содержит гомологий, то функиии $m$ u $f$ удовлетворяют условиям $(\forall x \in F)$

$$
\begin{gathered}
m(m(x))=x, \quad f(f(x))=\frac{1}{9} x \\
m(f(x))=-\frac{1}{3} m(x)-f(x)-\frac{1}{3} x, \quad f(m(x))=\frac{1}{3} m(x)+f(x)-\frac{1}{3} x .
\end{gathered}
$$

Д о к а з а т е л ь с т в о. Аналогично доказательству теоремы 2 рассмотрим случаи для $p>3$, выделенные в лемме.

При $(A, B)=(L, M)$ коллинеация $\gamma=\tau \sigma$ является гомологией с осью $[0,0]$ и центром $(\infty)$, поэтому матрица $L M=\left(\begin{array}{rr}-1 / 2 & -1 / 2 \\ 3 / 2 & -1 / 2\end{array}\right)$ должна удовлетворять условию $\theta(v, u) L M \in R$ для всех $v, u \in F$. В силу замкнутости $R$ по сложению вместо $L M$ достаточно рассмотреть матрицу $N=2 L M+E$. Тогда при $v=0$ получим $\theta(0, u) N=\left(\begin{array}{cc}0 & -m(u) \\ 3 u & 0\end{array}\right)=\theta(3 u, 0)$, откуда $f(x)=$ $-m(x) / 3$ для всех $x$. Случай $u=0$ дает то же условие.

При $(A, B)=(M, L)$, аналогично, $\gamma$ является гомологией с осью [0] и центром $(0)$, матрица $N$ удовлетворяет условию $N \theta(v, u) \in R$ для всех $v, u$. Рассматривая отдельно случаи $v=0$ и $u=0$, получим условие $f(m(x))=m(f(x))=-x / 3$ для всех $x$. 
При $A=B=M$ проверяем выполнение условия (1.1). При $v=0$ получим

$$
M^{-1} \theta(0, u) M=\frac{1}{4}\left(\begin{array}{cc}
m(u)+3 u & m(u)-u \\
3 m(u)-3 u & 3 m(u)+u
\end{array}\right) \in R,
$$

из замкнутости регулярного множества по сложению следуют равенства

$$
m(3 m(u)+u)=m(u)+3 u, \quad f(3 m(u)-3 u)=m(u)-u .
$$

Аналогично, при $v=0$ условие (1.1) приводит к равенствам

$$
m(-v-3 f(v))=v+3 f(v), \quad f(-v+9 f(v))=v-f(v) .
$$

Преобразуя, получаем условия теоремы 3.

Дополнительно рассмотрим ситуацию, когда коллинеация $\gamma$ является гомологией с осью $[\infty]$ и центром $(0,0)$. Тогда матрица $L M$ централизует $R$, поэтому матрица $N=2 L M+E$ также удовлетворяет условию $N \theta(v, u)=\theta(v, u) N$ для всех $v, u, f(v)=-v / 3, m(u)=u$,

$$
|\theta(v, u)|=\left|\begin{array}{cc}
u & -\frac{1}{3} v \\
v & u
\end{array}\right|=\frac{u^{2}}{3}\left(3+\left(\frac{v}{u}\right)^{2}\right) .
$$

Если $p-3$ не является квадратом, то $|\theta(v, u)| \neq 0$ для всякой ненулевой матрицы, и плоскость $\pi$ дезаргова.

Теорема 3 доказана.

Рассматривая произведение гомологий

$$
\left(\begin{array}{cc}
E & 0 \\
0 & L M
\end{array}\right) \quad \text { и } \quad\left(\begin{array}{cc}
L M & 0 \\
0 & E
\end{array}\right)
$$

приходим к очевидному следствию теоремы 3.

Следствие. Пусть $\pi$ - полуполевая плоскость порядка $p^{2 n}(p=2$ или $p>3-$ простое число) с ядром $\supseteq G F\left(p^{n}\right)$, ее группа линейнъх автотопизмов содержит подгруппь

$$
H_{1}=\left\langle\tau, \gamma_{1}\right\rangle \simeq S_{3}, \quad H_{2}\left\langle\tau, \gamma_{2}\right\rangle \simeq S_{3}
$$

где $\tau$ - бэровская инволюиия; $\gamma_{1}$ - гомология порядка 3 с осъю $[0,0]$ и иентром $(\infty) ; \gamma_{2}-$ гомология порядка 3 с осъю [0] и чентром (0). Тогда подгруппа $H_{3}=\left\langle\tau, \gamma_{1} \gamma_{2}\right\rangle$ такэне изоморфна $S_{3}$ и не содержит гомологий.

\section{4. Примеры}

Заметим, что указание матричного представления регулярного множества с достаточно общими ограничениями не является достаточным условием существования плоскости трансляций с таким регулярным множеством. Так, например, существование полуполевых плоскостей ранга 2 с регулярным множеством (2.1), допускающих бэровскую инволюцию (см. [4]), было подтверждено только в 1990 г. Хуангом и Джонсоном, построившими примеры восьми полуполевых плоскостей порядка 64 (см. [12]). Перечисление любых условий на группу автотопизмов приводит к естественному вопросу: существуют ли проективные плоскости при таких условиях? Покажем, что множество недезарговых полуполевых плоскостей, описанное теоремами 1 и 3, не пусто, т. е. найдем примеры таких плоскостей минимального возможного порядка. Напомним, что всякая аддитивная функция $g(x)$ на $G F\left(p^{n}\right)$ согласно [11] имеет вид

$$
g(x)=c_{0} x+c_{1} x^{p}+c_{1} x^{p^{2}}+\cdots+c_{n-1} x^{p^{n-1}}, \quad c_{0}, c_{1}, c_{2}, \ldots, c_{n-1} \in G F\left(p^{n}\right) .
$$


П р и м е p 1. Пусть $p=2, p^{2 n}=16, F=\{0,1, \alpha, \alpha+1\}, \alpha^{2}=\alpha+1$. Известно, что существуют ровно две неизоморфные недезарговы полуполевые плоскости порядка 16 (см., например, [5]). Покажем, что одна из них дает необходимый пример. Рассмотрим условия в теореме 1 , полагая

$$
m(x)=m_{0}\left(x+x^{2}\right), \quad f(x)=f_{0} x+f_{1} x^{2} \quad(x \in F), \quad \text { где } m_{0}, f_{0}, f_{1} \in F .
$$

Достаточным условием существования полуполевой плоскости, удовлетворяющей теореме 1, является требование

$$
\left|\begin{array}{cc}
u+v+m_{0}\left(v+v^{2}\right) & f_{0} v+f_{1} v^{2}+m_{0}\left(u+u^{2}\right) \\
v & u
\end{array}\right| \neq 0 \quad \forall u, v \in F,(u, v) \neq(0,0) .
$$

Перебор коэффициентов $m_{0}, f_{0}, f_{1}$, удовлетворяющих условиям теоремы 1 и (4.1), приводит к результатам, перечисленным в табл. 1.

Таким образом, найдены четыре полуполевые плоскости порядка 16 с регулярными множествами $R_{1}, R_{2}, R_{3}, R_{4}$, группа линейных автотопизмов которых содержит подгруппу, изоморфную $S_{3}$. Отметим, что эти плоскости изоморфны. Действительно, автоморфизм $x \rightarrow x^{2}$ поля $F$ переводит $R_{3}$ в $R_{4}$, и непосредственная проверка показывает, что выполнены равенства

$$
\left(\begin{array}{cc}
1 & \alpha \\
0 & 1
\end{array}\right) R_{1}\left(\begin{array}{cc}
1 & \alpha \\
0 & 1
\end{array}\right)=R_{2}, \quad\left(\begin{array}{cc}
0 & \alpha^{2} \\
\alpha^{2} & 1
\end{array}\right) R_{1}=R_{3}
$$

П р и м е р 2. Построим примеры плоскостей порядка 625, используя результаты теоремы 3. Рассмотрим поле $G F(25)=G F\left(5^{2}\right)$ как фактор-кольцо кольца $\mathbb{Z}_{5}[x]$ по идеалу, порожденному неприводимым в $\mathbb{Z}_{5}[x]$ многочленом $x^{2}-2$,

$$
G F(25) \simeq \mathbb{Z}_{5}[x] /\left(x^{2}-2\right)=\{0,1,2,3,4, \alpha, \alpha+1, \ldots, 4 \alpha+4\}, \quad \text { где } \alpha^{2}=2 .
$$

Т а б ли и ц а 1

\begin{tabular}{|c|c|c|c|c|}
\hline Регулярное множество & $m_{0}$ & $f_{0}$ & $f_{1}$ & Случай теоремы 1 \\
\hline$R_{1}$ & 1 & 1 & 0 & 1 \\
$R_{2}$ & 1 & 0 & 1 & 2 \\
$R_{3}$ & $\alpha$ & 1 & 0 & $1,2,4$ \\
$R_{4}$ & $\alpha+1$ & 1 & 0 & $1,2,4$ \\
\hline
\end{tabular}

Т а б ли и ц а 2

\begin{tabular}{|c|c|c|c|c|c|}
\hline № & $m_{0}$ & $m_{1}$ & $f_{0}$ & $f_{1}$ & Случай теоремы 3 \\
\hline 1 & 2 & 4 & 1 & 2 & 1 \\
2 & 4 & 2 & 2 & 1 & 1 \\
3 & $\alpha+4$ & $4 \alpha+2$ & $3 \alpha+2$ & $2 \alpha+1$ & 1 \\
4 & $2 \alpha+1$ & $3 \alpha$ & $\alpha+3$ & $4 \alpha$ & 1 \\
5 & $2 \alpha+2$ & $3 \alpha+4$ & $\alpha+1$ & $4 \alpha+2$ & 1 \\
6 & 2 & 4 & 2 & 1 & 2 \\
7 & 4 & 2 & 1 & 2 & 2 \\
8 & $\alpha+4$ & $4 \alpha+2$ & $\alpha+1$ & $4 \alpha+2$ & 2 \\
9 & $2 \alpha+1$ & $3 \alpha$ & $4 \alpha+3$ & $\alpha$ & 2 \\
10 & $2 \alpha+2$ & $3 \alpha+4$ & $3 \alpha+2$ & $2 \alpha+1$ & 2 \\
11 & $\alpha$ & $4 \alpha+1$ & $2 \alpha$ & 4 & 4 \\
12 & $2 \alpha$ & $3 \alpha+1$ & $4 \alpha$ & $3 \alpha$ & 4 \\
13 & $\alpha$ & $4 \alpha+1$ & $3 \alpha$ & $2 \alpha+3$ & $1,2,4$ \\
14 & $\alpha+4$ & $4 \alpha+2$ & $3 \alpha+2$ & $2 \alpha+1$ & $1,2,4$ \\
\hline
\end{tabular}


Рассмотрим условия в теореме 3, полагая

$$
m(x)=m_{0} x+m_{1} x^{5}, \quad f(x)=f_{0} x+f_{1} x^{5} \quad(x \in F), \quad \text { где } m_{0}, m_{1}, f_{0}, f_{1} \in F .
$$

Достаточным условием существования полуполевой плоскости, удовлетворяющей теореме 3, является требование

$$
\left|\begin{array}{cc}
m_{0} u+m_{1} u^{5} & f_{0} v+f_{1} v^{5} \\
v & u
\end{array}\right| \neq 0 \quad \forall u, v \in F, \quad(u, v) \neq(0,0) .
$$

Компьютерный перебор коэффициентов $m_{0}, m_{1}, f_{0}, f_{1}$, удовлетворяющих условиям теоремы 3 и (4.2), приводит к результатам, перечисленным в табл. 2. В таблице исключены изоморфные копии, полученные автоморфизмом $x \rightarrow x^{5}$ поля $F$.

Дальнейшее выделение в приведенном списке попарно изоморфных полуполевых плоскостей не проводилось, поскольку значительно более трудоемко в сравнении со случаем $|\pi|=16$. Существование полуполевых плоскостей порядка 625 с подгруппой линейных автотопизмов, изоморфной $S_{3}$, подтверждено.

В заключение следует заметить, что использованный метод может быть обобщен на случай полуполевых плоскостей ранга более 2. Доказанные результаты и найденные примеры могут быть использованы при дальнейшем исследовании полуполевых плоскостей с ограничениями на группу коллинеаций.

\section{СПИСОК ЛИТЕРАТУРЫ}

1. Мазуров В.Д., Хухро Е.И. Нерешенные вопросы теории групп. Коуровская тетрадь: изд. 16-е, доп., включающее архив решенных задач / Ин-т математики им. С. Л. Соболева СО РАН. Новосибирск, 2006. 193 с.

2. Подуфалов Н.Д., Дураков Б.К., Кравцова О.В., Дураков Е.Б. О полуполевых плоскостях порядка $16^{2} / /$ Сиб. мат. журн. 1996. Т. 37, № 3. С. 616-623.

3. Jha V., Johnson N.L. The translation planes of order 81 admitting $S L(2,5) / /$ Note di Matematica. 2005. Vol. 24, no. 2. P. 59-73. doi: 10.1285/i15900932v24n2p59.

4. Biliotti M., Jha V., Johnson N.L., Menichetti G. A structure theory for two-dimensional translation planes of order $q^{2}$ that admit collineation group of order $q^{2} / /$ Geom. Dedicata. 1989. Vol. 29. P. 7-43. doi: 10.1007/BF00147468.

5. Levchuk V.M., Kravtsova O.V. Problems on structure of finite quasifelds and projective translation planes // Lobachevskii J. Math. 2017. Vol. 38, no 4. P. 688-698. doi: 10.1134/S1995080217040138.

6. Rúa I.F., Combarro E.F., Ranilla J. Classification of semifields of order 64 // J. Algebra, 2009, vol. 322, no. 11, pp. 4011-4029. doi: 10.1016/j.jalgebra.2009.02.020.

7. Кравцова О.В., Моисеенкова Т.В. Полуполевые проективные плоскости, допускающие подгруппу коллинеаций, изоморфную $S_{3}$. // Алгебра, теория чисел и дискретная геометрия: современные проблемы, приложения и проблемы истории: материалы Междунар. конф. посвященной 80-летию со дня рождения профессора Мишеля Деза. Тула: Изд-во Тульского гос. пед. ун-та им. Л. Н. Толстого, 2019. С. 260-262.

8. Hughes D.R., Piper F.C. Projective planes. N Y: Springer-Verlag, 1973. 292 p.

9. Johnson N.L., Jha V., and Biliotti M. Handbook of finite translation planes. Boca Raton; London; N Y: Chapman and Hall, 2007. 861 p.

10. Кравцова О.В. Полуполевые плоскости, допускающие бэровскую инволюцию // Изв. Иркут. гос. ун-та. Сер. Математика. 2013. №2. С. 26-38.

11. Vaughan, T.P. Polynomials and linear transformations over finite fields // J. Reine Angew. Math. 1974. Vol. 267. P. 179-206. doi: 10.1515/crll.1974.267.179.

12. Huang H. , Johnson N.L. 8 semifield planes of order $8^{2} / /$ Discrete Math. 1990. Vol. 80, no. 1. P. 69-79. doi: 10.1016/0012-365X(90)90296-T . 
Кравцова Ольга Вадимовна

канд. физ.-мат. наук, доцент

доцент кафедры высшей математики № 2

Сибирский федеральный университет, г. Красноярск

e-mail: ol71@bk.ru

Моисеенкова Татьяна Владимировна

канд. физ.-мат. наук

доцент кафедры высшей математики № 2

Сибирский федеральный университет, г. Красноярск

e-mail: tanya-mois11@yandex.ru

\section{REFERENCES}

1. Mazurov V.D. and Khukhro E.I. (eds.). The Kourovka Notebook: Unsolved Problems in Group Theory, 16th ed., Novosibirsk: Sobolev Inst. Math. Publ., 2006, 195 p.

2. Podufalov N.D., Durakov B.K., Kravtsova O.V., Durakov E.B. On semifield planes of order $16^{2}$. Siberian Math. J., 1996, vol. 37, no. 3, pp. 535-541. doi: 10.1007/BF02104857.

3. Jha V., Johnson N.L. The translation planes of order 81 admitting $S L(2,5)$. Note di Matematica, 2005, vol. 24, no. 2, pp. 59-73. doi: 10.1285/i15900932v24n2p59 .

4. Biliotti M., Jha V., Johnson N.L., Menichetti G. A structure theory for two-dimensional translation planes of order $q^{2}$ that admit collineation group of order $q^{2}$. Geom. Dedicata, 1989, vol. 29, pp. 7-43. doi: $10.1007 / \mathrm{BF} 00147468$.

5. Levchuk V.M., Kravtsova O.V. Problems on structure of finite quasifields and projective translation planes. Lobachevskii J. Math., vol. 38, no. 4, pp. 688-698. doi: 10.1134/S1995080217040138.

6. Rúa I.F., Combarro E.F., Ranilla J. Classification of semifields of order 64. J. of Algebra, 2009, vol. 322, no. 11, pp. 4011-4029. doi: 10.1016/j.jalgebra.2009.02.020 .

7. Kravtsova O.V., Moiseenkova T.V. Semifield projective plane that admit collineation subgroup isomorphic to $S_{3}$. In: XVI International Conference "Algebra, Number Theory and Discrete Geometry: modern problems and applications", dedicated to the 80th anniversary of the birth of professor Michel Desa, Tula: Tula State Pedagogical University of Leo Tolstoy Publ., 2019, pp. 260-262.

8. Hughes D.R., Piper F.C. Projective planes. New York: Springer-Verlag, 1973, 292 p. ISBN: 9780387900445 .

9. Johnson N.L., Jha V., and Biliotti M. Handbook of finite translation planes. Boca Raton; London; N Y: Chapman and Hall, 2007, 861 p. ISBN: 1-58488-605-6/hbk

10. Kravtsova O.V. Semifield planes of even order that admit the baer involution. The Bulletin of Irkutsk State University. Ser. Mathematics, 2013, vol. 6, no. 2, pp. 26-37 (in Russian).

11. Vaughan T.P. Polynomials and linear transformations over finite fields. J. Reine Angew. Math., 1974, vol. 267, pp. 179-206. doi: 10.1515/crll.1974.267.179.

12. Huang H., Johnson N.L. 8 semifield planes of order $8^{2}$. Discrete Math., 1990, vol. 80, no. 1, pp. 69-79. doi: 10.1016/0012-365X(90)90296-T .

Received July 25, 2019

Revised October 7, 2019

Accepted October 14, 2019

Funding Agency: This work was supported by the Russian Foundation for Basic Research (project no. 19-01-00566 A).

Olga Vadimovna Kravtsova, Cand. Sci. (Phys.-Math.), Siberian Federal University, Krasnoyarsk, 660041 Russia, e-mail: ol71@bk.ru.

Tatyana Vladimirovna Moiseenkova, Cand. Sci. (Phys.-Math.), Siberian Federal University, Krasnoyarsk, 660041 Russia, e-mail: tanya-mois11@yandex.ru .

Cite this article as: O.V.Kravtsova, T.V. Moiseenkova. Semifield planes of rank 2 admitting the group $S_{3}$, Trudy Instituta Matematiki i Mekhaniki URO RAN, 2019, vol. 25, no. 4, pp. 118-128. 